\title{
Integration of Smart Antenna System in Mobile Ad Hoc Networks
}

\author{
Mohammed A. Abdala and Areej K. Al-Zuhairy
}

\begin{abstract}
The capacity of a Mobile Ad-hoc Network (MANET) can be severely limited due to interference constraints. One way of improving the overall capacity of ad-hoc networks is by the use of smart antenna system (SAS). SAS has the advantage over traditional omnidirectional antennas of being able to orientate radio signals into the concerned directions in either transmission mode or in reception mode. The omnidirectional antennas in broadcasting over the whole network are the source of an excessive redundancy of broadcast packet receptions within each node. The SAS consist of a uniform linear array (ULA) and the signal processing unit, employing constant modulus algorithm (CMA) is implemented using MATLAB R2012a. For the MANET we used geographic routing protocol (GRP) which is Optimum Network Engineering Tool's (OPNET's) custom model routing protocol where each node uses the global positioning system (GPS) to identify its own position and implemented in OPNET modeler 14.5. A co-simulation is then made between OPNET modeler 14.5 and MATLAB R2012a to take advantage of SAS and uses it with MANET in OPNET 14.5. Using this approach, exploring SAS for MANET improves throughput and Signal to Noise Ratio overall the network and decreases Bit Error Rate compared to omnidirectional antenna.
\end{abstract}

Index Terms-CMA, mobile Ad Hoc network, omnidirectional antenna, smart antenna system.

\section{INTRODUCTION}

The earliest of MANETs were called "packet-radio" networks, first sponsored by the United States (U.S.) Defense Advanced Research Projects Agency (DARPA) in the early 1970s. It is interesting to note that some early packet-radio systems predated the Internet and, indeed, were part of the motivation of the original Internet protocol (IP) suite. Later DARPA experiments included the Survivable Radio Network (SURAN) project, which took place in the 1980s. The third wave of academic activity on Wireless Ad-Hoc Networks started in the 1990s, especially with the wide usage of inexpensive 802.11 radio cards for personal computers [1].

MANET is a self-configurable, Infrastructure less, autonomous and self-healing system of nodes using wireless links. MANETs fall into the category of wireless networks in which each device can act as a source, destination and a moving router and can communicates with other devices in its range [2]. The advantages of MANETs lie in their low costs (because no infrastructure is required) and high flexibility. The drawbacks include reduced efficiency, smaller communication range, and restrictions on the number

Manuscript received May 13, 2013; revised July 2, 2013

The authors are with College of Information Engineering, Al-Nahrain University, Baghdad, Iraq (e-mail: dr.maabdala@ieee.org, areejalzuhairy@gmail.com). of devices that can be included in a network. Ad-Hoc Networks play a major role in the recent proliferation of sensor networks, which allow communications between machines for the purpose of building control, factory automation, surveillance, etc. and also play a role in emergency communications (when infrastructure was destroyed, e.g., by an earthquake) as well as military communications [3]. The routing in MANETs is very challenging due to the frequent updates for changes in topologies, and active routes may be disconnected as mobile nodes move from one place to another. Routing schemes in MANETs must include mechanisms that cope with difficulties incurred by node mobility and topology changes along with lower consumption of energy, communications bandwidth, and computing resources [4]. The routing protocols in MANETs can hence be divided into five categories [5]: Source-initiated (reactive or on-demand) Table-driven (proactive), Hybrid, Location-aware (geographical) and Multipath.

Conventionally, MANETs have been known to use omnidirectional antennas for transmission as well as reception. The use of omnidirectional antennas may result in lower power efficiency due to interference caused by the transmission of packets in undesired directions. Use of SAS in MANETs is envisioned to take advantage of Space Division Multiple Accesses (SDMA) to increase network efficiency by directing the transmitted power in the desired direction [6].SAS allows the energy to be transmitted or received in a particular direction as opposed to disseminating energy in all directions; this helps in achieving significant spatial re-use and thereby increasing the capacity of the network [7]. For many years, the researchers concentrate on analyzing the performance of SAS in MANET by proposing different Medium Access Control (MAC) protocols and how estimating Direction of Arrival (DOA) by using several Conventional Techniques, However, these studies and the proposed techniques did not take into account position-based (geographic) routing protocol in OPNET modeler 14.5 that use GPS to estimate node positions and find DOA between mobile nodes in MANET. In [8] the authors propose a MAC protocol for smart antenna used networks. In [9] the authors present a tool for the modeling, analysis and simulation of DOA estimation and adaptive beamforming needed in the design of SAS Systems. In [10] the authors propose a novel Location-Enhanced On-Demand (LEOD) Routing protocol based on smart antenna technique. The model is implemented on MANET with SAS and using GRP. The radio receiver parameters such as Throughput, Signal to Noise Ratio (SNR) and Bit Error Rate (BER) are used to investigate the performance of SAS in MANET with 802.11g.

This paper is organized as follows: In Section II, Mobile 
Ad-Hoc Network (MANET). In Section III, Smart Antenna System. In Section IV, Co-simulation between OPNET and MATLAB for MANET with smart antenna. In Section V, network layout and simulation parameters. In Section VI, performance metrics. In Section VII, results and Section VIII, conclusion.

\section{Mobile Ad Hoc Networks}

A MANET is an autonomous ad-hoc wireless networking system consisting of independent nodes that move dynamically changing network connectivity. Unlike cellular wireless networks, no static or fixed infrastructureless exists in MANET, and no centralized control can be available. The network can be formed anywhere, at any time, as long as two or more nodes are connected and communicate with one another either directly when they are in radio range of each other or via intermediate mobile nodes because of flexibility that a MANET offers [4]. In contrast, cellular networks are managed by a centralized administration or Base Station Controller (BSC) where each node is connected to a fixed base station. Moreover, cellular networks provide single hop connectivity between a node and a fixed base station while MANETs provide multihop connectivity between Nodes A (source) and Node B (destination), as illustrated in Fig. 1.This figure shows an example of two nodes, Node A and Node B, that desire to exchange data and at some distance apart. Because they are out of radio range with each other, it is necessary for Node $\mathrm{A}$ to use the neighboring or intermediate nodes in forwarding its data packets to Node B [11].

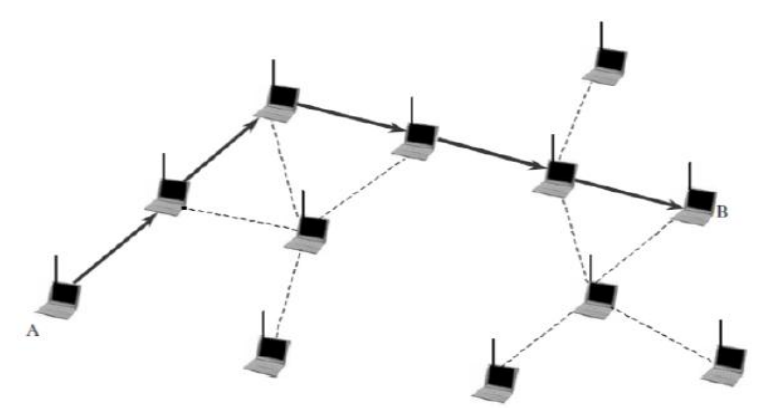

Fig. 1. Multihop Example of MANET.

The routing in MANETs is very challenging due to the frequent updates for changes in topologies, and active routes may be disconnected as mobile nodes move from one place to another. Routing protocols can be classified as follows: unicast, broadcast, multicast, and geocast [11]. There are some other routing protocols that do not rely on any traditional routing mechanisms, instead rely on the location awareness of the participating nodes in the network. Generally, in traditional MANETs, the nodes are addressed only with their IP addresses. But, in case of location-aware routing mechanisms, the nodes are often aware of their exact physical locations in the three-dimensional world. This capability might be introduced in the nodes using GPS or with any other geometric methods. GPS is a worldwide, satellite-based radio navigation system that consists of 24 satellites in six orbital planes. By connecting to the GPS receiver, a mobile node can know its current physical location [12].GRP also known as position based routing, is a well-researched approach for ad hoc routing. GRP is based on two assumptions; nodes are aware of their own geographic locations and also of its immediate neighbors and source node are aware of position of destination. The nodes update its immediate neighbor's locations periodically by beaconing. The data packets are routed through the network using the geographic location of the destination and not the network address. GRP operates without routing tables and routing to destination depends upon the information each node has about its neighbors [13]. In the position (location)-based routing protocols, a mobile node uses a directional antenna or GPS system to estimate its $(x, y)$ position. If GPS is used, every node knows it's $(x, y)$ position assuming $z=0$. Fig. 2, Shows two mobile nodes with their positions determined using GPS The positions of the two mobile nodes in Fig. 2, are $\left(x_{1}, y_{1}\right)$ and $\left(x_{2}, y_{2}\right)$ respectively. Using Fig. 2. The distance (d) between the two Mobile nodes and the angle of arrival $(\theta)$ can be calculated as in (1) and (2) respectively [14].

$$
\begin{gathered}
d=\sqrt{\left(x_{2}-x_{1}\right)^{2}+\left(y_{2}-y_{1}\right)^{2}} \\
\theta=\tan ^{-1} \frac{y_{2}-y_{1}}{x_{2}-x_{1}}
\end{gathered}
$$

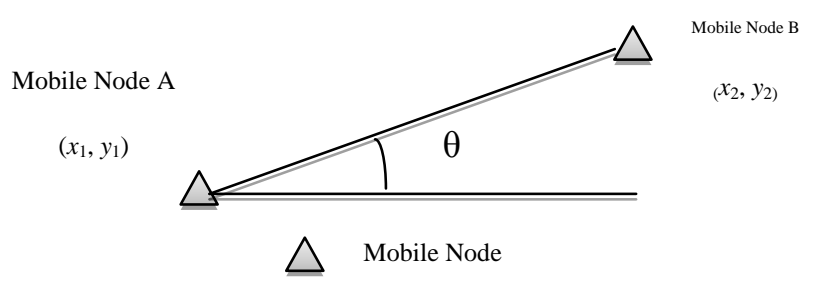

Fig. 2. Position-based routing protocol that uses GPS to determine mobile nodes $(x, y)$ positions

\section{SMART ANTENNA SySTEM}

SAS is a subsystem that contains multiple antennas; based on the spatial diversity and signal processing, it significantly increases the performance of wireless communication systems as shown in Fig. 3 Direction finding and beamforming are the two most fundamental topics of SAS Direction finding is used to estimate the number of emitting sources and D.O.A., while beamforming is used to estimate Signal of Interest (SOI) in the presence of interference [15] The main feature of SAS is the selection of smart algorithms in adaptive array. Using beamforming algorithms the weight of antenna arrays can be adjusted to form certain amount of adaptive beam or narrow beam to track the corresponding users automatically. At the same time SAS minimizes interference arising from other users by introducing nulls in their directions, and the desired signals can be extracted. The weight adaptation is the "smart" part of the SAS

The most important benefit of SAS is achieving higher network capacity, it increase network capacity by precise control of signal nulls quality and mitigation of interference.

The SAS is a new technology and has been applied to the mobile communication system such as GSM and CDMA. not balance dimensionally. If you must use mixed units, clearly state the units for each quantity in an equation.

SAS can be considered to achieve increased revenues for network operators and give customers less blocked or 
dropped calls [16]. SAS, however, are usually categorized as switched-beam and adaptive-array systems. Although both systems attempt to increase gain in the direction of the user, only the adaptive-array system offers optimal gain, while simultaneously identifying, tracking, and minimizing interfering signals [17].

Beam forming is controlling 'directionality of' or 'sensitivity to' particular radiation pattern. Adaptive beamforming can be done in many ways. The technology combines the inputs of multiple antennas (from an antenna array) to form very narrow beams toward individual user.

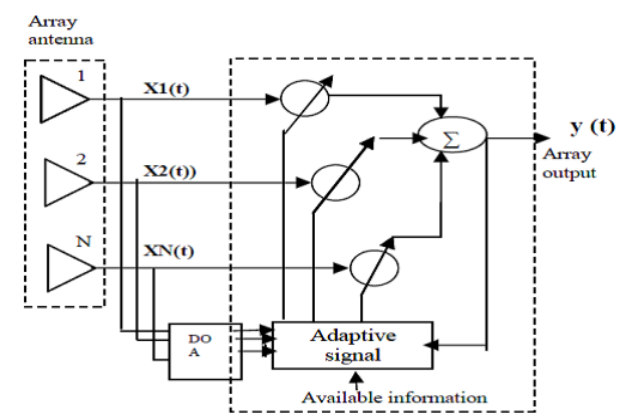

Fig. 3. Functional Block diagram of Smart antenna system [9].

A generic adaptive beamformer is shown in Fig. 4. The weight vector $W(n)$ is calculated using the signal $x(n)$ received by multiple antennas. An adaptive processor will minimize the error $e(n)$ between a desired signal $d(n)$ and the array output $Y(n)[16]$.

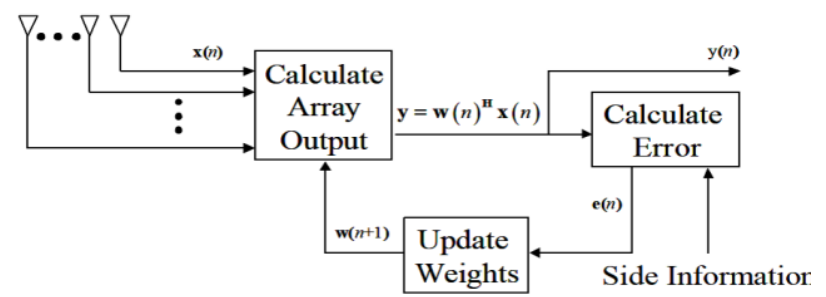

Fig. 4. Adaptive Antenna Array [18].

The CMA adaptive beamforming is a gradient-based algorithm that works on the theory that the existence of interference causes changes in the amplitude of the transmitted signal, which otherwise has a constant envelope [16]. In general, the CMA algorithm seeks a beamformer weight vector that minimizes a cost function:

$$
J_{p, q}=\varepsilon\left\{\left.|| y(n)\right|^{p}-\left.1\right|^{q}\right\}
$$

The weight is updated:

$$
w(n+1)=w(n)-\mu \nabla_{w, w *}\left(J_{p, q}\right)
$$

where the step-size parameter has been denoted by $\mu$.

The gradient vector is given by:

$$
\nabla_{w, w *\left(\hat{J}_{1,2}\right)}=\frac{\partial J 1,2}{\partial w *}=\varepsilon\left[x(n)\left(y(n)-\frac{y(n)}{|y(n)|}\right)^{*}\right]
$$

And therefore, the resulting weight vector:

$$
\begin{aligned}
& w(n+1)=w(n)-\mu\left[y(n)-\frac{y(n)}{|y(n)|}\right]^{*} x(n)=w(n)+ \\
& \mu e^{*}(n) x(n)
\end{aligned}
$$

where

$$
e(n)=y(n) /|y(n)|-y(n)
$$

The term $\left(\frac{y(n)}{|y(n)|}\right)$ in CMA plays the same role as the desired signal $d(n)$ in theleast mean square algorithm (L.M.S.).

\section{Co-Simulation OPNET AND MATLAB FOR MANET With SMART ANTENNA}

Most systems consider the use of omnidirectional antennas for ad hoc networks. However, this reserves the spectrum over a large area, wasting network resources. SAS not only can mitigate this problem, but also can provide the other advantages as shown above. Since SAS is a physical-layer technique, can be used with modified MAC/routing in ad hoc to achieve the full benefits of SAS [16].OPNET provides a very stable discrete event simulation engine while MATLAB, is software for numerical calculations and provides communication engineers with a vast library forimplementing communication systems such as channel models and adaptive beamforming algorithms. By integrating MATLAB simulation with OPNET we are able to reuse the algorithms developed in MATLAB and analyze their performance and effect on the upper layers (specifically, data link and network layers) of the communication system, which would be difficult to realize without a discrete event simulator. In this paper, we have interfaced MATLAB and OPNET so as to reuse the adaptive beamforming algorithm CMA, which was developed in MATLAB, and use the graphics library of MATLAB to provide the capability and observe the dynamic changes in the antenna patterns during simulation execution time as shown in Fig. 5.

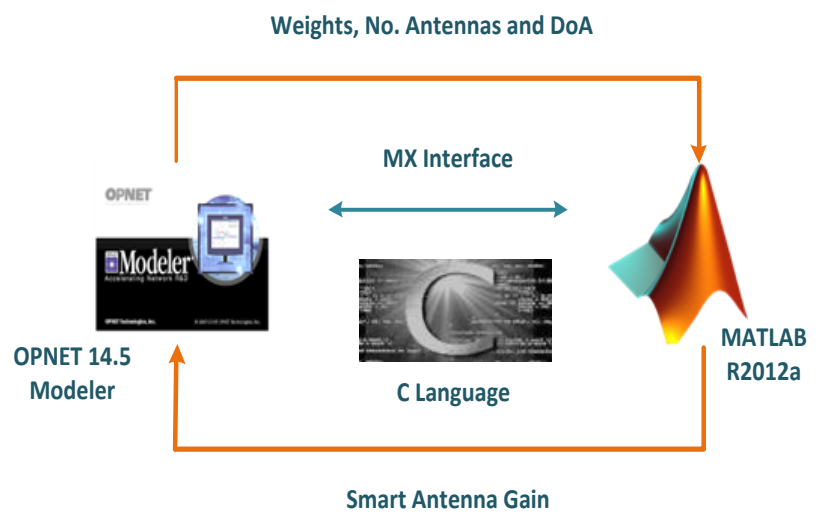

Fig. 5. OPNET and MATLAB Interface.

\section{NETWORK LAYOUT AND SIMULATION PARAMETERS}

Throughput, SNR and BER for the radio receiver are the performance metrics adopted to compare the two different scenarios. First scenario for simulating MANET with Omnidirectional antenna used GRP and voice application (low quality speech). The second scenario also for simulation MANET with SAS used GRP and voice application (low quality speech) but with SAS, The network layout for MANET scenario is shown in Fig. 6. And the simulation parameters used in OPNET and MATLAB are shown in Table I and Table II respectively. 


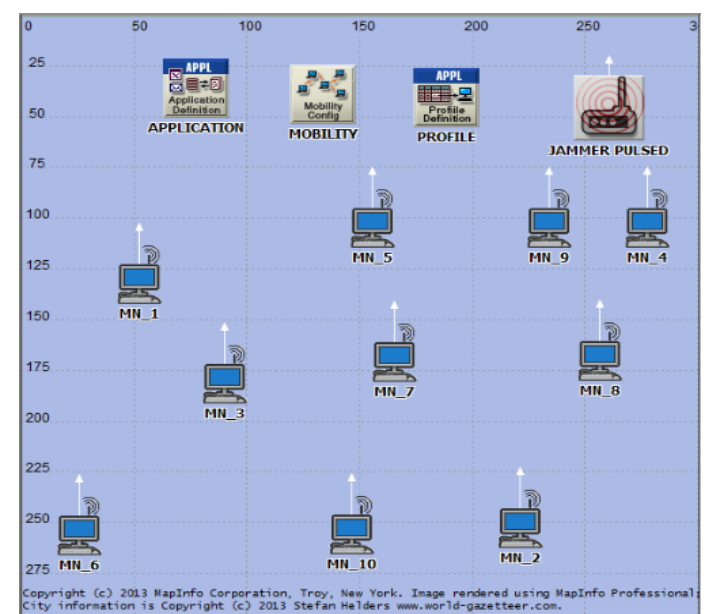

Fig. 6. Network layout for MANET scenario.

TABLE I: OPNET SIMULATION PARAMETERS

\begin{tabular}{ll}
\hline \hline Parameter & Value \\
\hline Simulation Time & 80 seconds \\
Environment size & $(300 * 300)$ square meter \\
No. of Nodes & Ten nodes \\
Physical characteristics & Extended rate PHY $(802.11 \mathrm{~g})$ \\
Data rate & 54 Mbps \\
Transmit power & 0.00033 Watt \\
AD-HOC routing & GRP \\
Protocol & \\
Trajectory Vector & Vector \\
Traffic type & Voice (low quality speech) \\
Ragain model & Omnidirectional(none),Smart(dra_ragain) \\
Mobility & Default Random waypoint \\
\hline
\end{tabular}

TABLE II: MATLAB SIMULATION PARAMETERS

\begin{tabular}{ll}
\hline \hline Parameter & Value \\
\hline Array type & ULA \\
No. of .antenna elements & 8 \\
Carrier frequency & $2.4 \mathrm{GHz}$ \\
Step size & 0.008 \\
Initial weights & {$[0.0010 .0010 .0010 .0010 .0010 .0010 .001$} \\
& $0.001]$ \\
DOA & Change within node mobility refer to (2) \\
\hline \hline
\end{tabular}

\section{PERFORMANCE METRICS}

In this section we make comparisons between performance of omnidirectional antenna MANET and with that of SAS according to three performance metrics Bit Error Rate (BER), Signal to Noise Ratio (SNR) and Throughput.

Bit Error Rate: the number of bit errors divided by the total number of transferred bits during a studied time interval. BER is a unitless performance measure, often expressed as a percentage.

Throughput: The ratio of the total data reaches the receiver from the sender. The time it consume by the receiver to receive the last packet is called throughput.

Signal to Noise Ratio: is the ratio of the average power of the information signal to the accumulated average power of all background and interference noise sources.

\section{RESULTS}

Fig. 7 shows BER values for the receiver mobile nodes (MN_2, MN_4 and MN_6). The figure shows that SAS decreases BER for MN_4 and MN_6 by 93.5 and $89.9 \%$ respectively. The bit error rate reveals that the bit error rate at the receiver node $\mathrm{MN} \_2$ was 0.0002 initially as the distance between the jammer node and receiver node MN_2 is large. However, after about 4 seconds, the direction vector between the jammer node and the receiver node was in line with the direction of greatest gain for the receiver antenna. Therefore, MN_2 started to receive interference from the jammer node and the peak value of BER at MN_2 approached 0.0023 errors/bits.

Fig. 8 shows SNR values for the receiver mobile nodes (MN_2, MN_4 and MN_6). The figure shows that SAS increases by $76.1 \%, 72.1 \%$ and $72.9 \%$ respectively compared to omnidirectional antenna.

Fig. 9 shows throughput values for the receiver mobile nodes (MN_2, MN_4 and MN_6). The figure shows that SAS increases by $98.1 \%, 92.6 \%$ and $97 \%$ respectively.

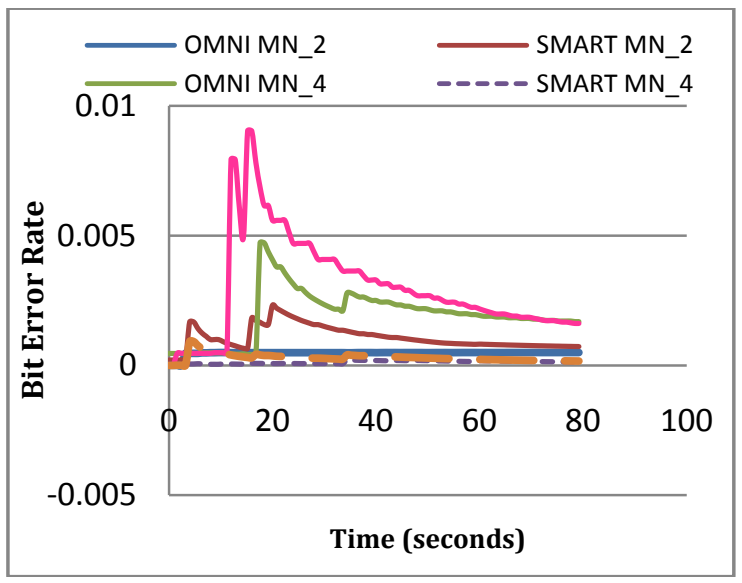

Fig. 7. Bit Error Rate statistics for the receiver mobile nodes.

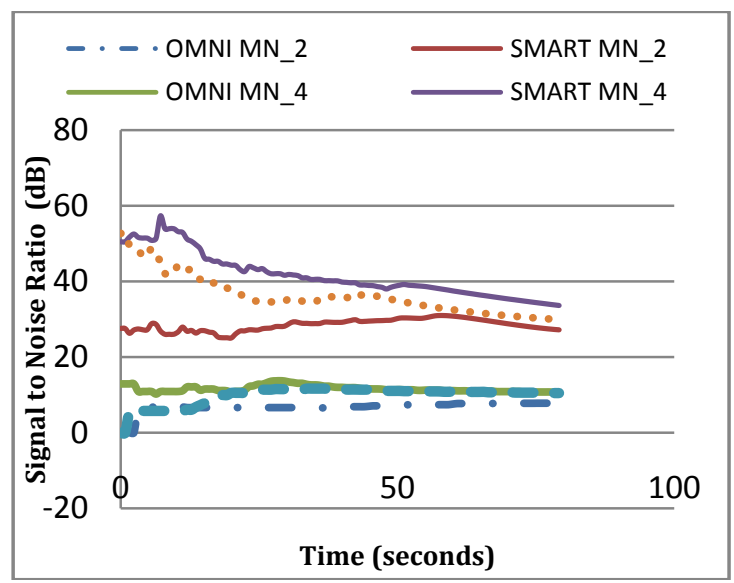

Fig. 8. Signal to Noise Ratio statistics for the receiver mobile nodes.

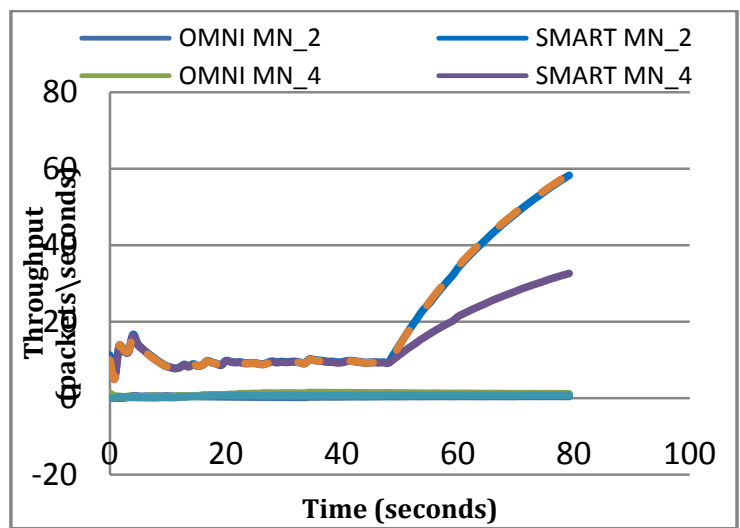

Fig. 9. Throughput statistics for the receiver mobile nodes. 


\section{COMPARISON WITH OTHER WORKS}

TABLE III: COPARISON BETWEEN OMNIDIRECTIONAL ANTENNA AND SAS

\begin{tabular}{|c|c|}
\hline Current Work & Related Work \\
\hline $\begin{array}{l}\text { BER is decreasedby } 91.7 \% \text { on } \\
\text { average in MN_4 and MN_6 but } \\
\text { MN_2 in SAS is higher than } \\
\text { omnidirectional antenna according } \\
\text { to reason mentioned above and } \\
\text { reaches to } 0.002 \text {. }\end{array}$ & $\begin{array}{l}\text { In [19] BER was tested for } \\
\text { different types of modulation the } \\
\text { maximum value reaches to } \\
\text { approximately } 10^{-1}\end{array}$ \\
\hline $\begin{array}{l}\text { SNR is increased up to } 73.7 \% \text { on } \\
\text { average in SAS as compared to } \\
\text { omnidirectional antenna. }\end{array}$ & $\begin{array}{l}\text { In [18]the average SINR is better } \\
\text { than for the omnidirectionalcase } \\
\text { which has the value } \\
\text { approximately of } 40 \mathrm{~dB} \text { at its peak } \\
\text { value. }\end{array}$ \\
\hline $\begin{array}{l}\text { Throughput in SAS shows an } \\
\text { improvement about } 95.9 \% \text { over } \\
\text { omnidirectional antenna. }\end{array}$ & $\begin{array}{l}\text { In }[20] \text { the results show that } \\
\text { throughput improved by } 164 \% \text {. }\end{array}$ \\
\hline
\end{tabular}

\section{CONCLUSION}

We have presented a methodology for implementing SASin OPNET simulation. The model supports Uniform Linear Arrays and allows adaptive beamforming algorithms CMA Through simulation results we observed that using SASprovides better Signal to Noise Ratio and throughput as compared to the scenarios using omnidirectional antenna BER generally decreases when employing SAS as compared to omnidirectional antenna except inMN_2 when direction vector between the jammer node and the receiver node was in line with the direction of greatest gain for the receiver antenna.

\section{REFERENCES}

[1] X. Y. Li, Wireless Ad Hoc and Sensor Networks Theory and Applications, United States: Cambridge University Press, 2008, ch. 1.

[2] S. Naseer, S. A. Hussain, I. Raza, S. R. Chaudry, J. S. Mirza, and M. H. Raza, "Mobile ad-hoc network routing protocols: A simulation and performance analysis using multimedia traffic," Journal of Basic and Applied Scientific Research, vol. 2, no. 10, pp. 9925-9930, 2012.

[3] A. F. Molisch, Wireless Communications, $2^{\text {nd }}$ ed. United Kingdom: Wiley, 2011, ch. 1

[4] R. R. Roy, Handbook of Mobile Ad Hoc Networks for Mobility Models, Springer Science+Business Media, LLC, 2011, ch. 1.

[5] A. Boukerche, Algorithms and Protocols for Wireless and Mobile Ad Hoc Networks, Hoboken, New Jersey: Wiley, 2009, ch. 5.

[6] J. Alphonse and M. N. M. Saad, "Optimizing the performance of MANET with an enhanced antenna positioning system," IJCSNS International Journal of Computer Science and Network Security, vol. 9, no.4, pp. 50-54, April 2009.

[7] P. Mohapatra and S. Krishnamurthy, Ad Hoc Networks Technologies and Protocols, United States of America: Springer Science + Business Media, Inc., Boston, 2005, ch. 7.

[8] J. Ma, H. Sekiya, N. Komuro, and S. Sakata, "MAC protocol for ad hoc network using smart antenna with pulse/tone exchange," in Proc. ICN 2013: The Twelfth International Conf. on Networks, IARIA, 2013.

[9] S. W.Varade and K. D. Kulat, "Robust algorithms for DOA estimation and adaptive beamforming for smart antenna application," in Proc. 2nd International Conf. on Emerging Trends in Engineering and Technology, ICETET, 2009.

[10] A. Quinteroa, D. Y. Lia, and H. Castro, "A location routing protocol based on smart antennas for ad hoc networks," Journal of Network and Computer Applications, vol. 30, pp. 614-636, 2007.

[11] C. A. Balanis, Antenna Theory Analysis and Design, Hoboken, New Jersey: John Wiley \& Sons, Inc., 2005, ch. 16.

[12] S. Misra, I. Woungang, and S. Misra, Guide to Wireless Ad Hoc Networks, Springer-Verlag London Limited, 2009, ch. 4.

[13] A. Tamizhselvi and Dr. R. S. D. W. Banu, "Performance evaluation of geographical routing protocol under different traffic scenario,"
International Journal of Computer Science and Telecommunications vol. 3, Issue 3, pp. 64-67, March 2012.

[14] M. Mikki, "Energy efficient location aided routing protocol for wireless MANETs," International Journal of Computer Science and Information Security, vol. 4, no. 1 \& 2, 2009.

[15] K. Du and M. N. S. Swamy, Wireless Communication Systems from RF Subsystems to $4 G$ Enabling Technologies, New York, the United States of America: Cambridge University Press, 2010, ch. 18.

[16] S. R. Inamdar, R. M. Yadahalli et al., "Smart antenna system using cross layer design in wireless ad hoc networks: Trends and challenges," World Journal of Science and Technology, vol. 2, no. 5, pp. 74-79, 2012.

[17] M. Chryssomallis, "Smart antennas," IEEE Antennas and Propagation Magazine, vol. 42, no. 3, June 2000.

[18] R. Mahajan, "Cross layer optimization: system design and simulation methodologies," M.S. thesis, Electrical Eng., Virginia Polytechnic Institute and State Univ., Blacksburg, VA, 2003.

[19] S. Bellofiore, J. Foutz, R. Govindarajula, I. Bahçeci, C. A. Balanis, A. S. Spanias, J. M. Capone, and T. M. Duman, "Smart antenna system analysis, integration and performance for mobile ad-hoc networks (MANETs)," IEEE Transactions on Antennas and Propagation, vol 50, no. 5, May 2002.

[20] R. Verma, A. Prakash, P. K. Verma, N. Tyagi, and R. Tripathi, "A novel MACA novel MAC protocol for MANETs using Smart Antenna System protocol for MANETs using smart antenna system," IEEE, 2010.

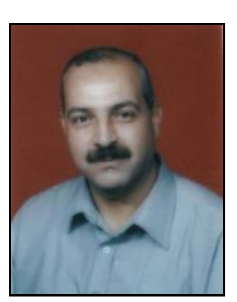

Mohammed A. Abdala was born in Baghdad, IRAQ in May 12, 1962. He obtained the doctor of philosophy (Ph.D.), Electronic EngineeringTransconductance \& Noise Analysis in GaAs MESFETs, Lancaster University, UK in Oct. 1991. $\mathrm{He}$ obtained the master of science (M.Sc.) in Semiconductor Devices, Lancaster University, UK in Oct. 1988, and the bachelor of science (B.Sc.) (Hons.) in Electrical \& Electronic Engineering. Grade: Distinction, University of Technology, Baghdad, Iraq

in June 1984.

He is the head of Networks Engineering Department at the Information Engineering College, Nahrain University, Baghdad, Iraq. He has 25 years of academic \& consulting experience in Microelectronics, Solid State Electronics, Software Engineering, Pattern Recognition and Verification. He published more than 20 scientific \& technical papers. He currently does the teaches \& conducts research programs in the areas of image processing, Genetic and PSO Optimization Algorithms, pattern recognition \& verification, VHDL implementation of image compression algorithms, software engineering and e-commerce, wireless sensor networks, wireless Ad Hoc Networks and others. He supervised over 50 postgraduate theses and over 80 graduate projects and examined over 90 M.Sc. \& Ph.D. theses.

Associate professor Dr. Abdala is a member of IEEE \& Iraqi Engineers Union and consultant to several state companies specialized in manufacturing of computers \& electronic components, devices, and systems. He is active in works that led to the establishment of several engineering departments \& colleges in Iraq. He is a member of the Editorial Board of i-manager's Journal on Information Technology (JIT) in India and the Information and Communication Technology Journal in Al-Nahrain University in Iraq. He is a PC member and reviewer for many worldwide conferences. He is a referee for the publication of scientific papers in several journals and organized many lectures \& training courses in the field of solid-state electronics, LCA technology, ASIC design and Software Engineering Methodologies for engineers.

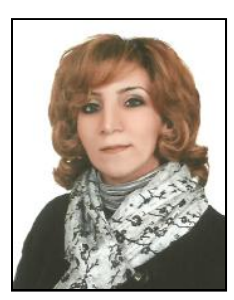

Areej K. Al-Zuhairy was born in Baghdad, IRAQ in January 17, 1984. She is Currently a master of science (MSc.) candidate in Information and Communication Engineering, Al-Nahrain university, Baghdad, Iraq. She obtained the bachelor of science (B.Sc.) in Computer Engineering and Information Technology university, Technology University, Baghdad, Iraq in 2005.

Areej K. Al-Zuhairy is a M.Sc. candidate in Information and Communication Engineering at the Information Engineering College, Nahrain University, Baghdad, Iraq. 